\title{
The Renal Transplant Patient-Updates for the Pediatrician
}

\section{Margret Bock, MD, MS Jens Goebel, MD}

\begin{abstract}
Address
*Pediatric Nephrology, Children's Hospital Colorado, 13123 East 16th Ave, 328, Aurora, C0, 80045, USA

Email: Margret.Bock@childrenscolorado.org

Published online: 19 April 2016

(C) Springer International Publishing AG 2016

This article is part of the Topical Collection on Pediatric Nephrology

Keywords Kidney transplant · BK virus - Rejection - Immunosuppression - Cardiovascular disease obesity

\section{Opinion statement}

Kidney transplantation remains superior to chronic dialysis as the treatment option for end-stage renal disease (ESRD) in children and adults. Recent refinements in pediatric kidney transplantation have mainly sharpened our focus on the major cardiovascular (CV) morbidity risk for children with kidney transplants, unfortunately including our recognition of how poorly we are managing this risk, and on new approaches to gauge and manage immunological risk, specifically monitoring our patients for evidence of too much (BK virus) or not enough (donor-specific antibodies, DSAs, and antibody-mediated rejection, AMR) immunosuppression.
\end{abstract}

\section{Introduction}

\section{Current state of kidney transplantation in children} Kidney transplantation is the treatment of choice for children with end-stage renal disease (ESRD) with numerous studies demonstrating advantages of transplantation over dialysis. Dialysis and ESRD are associated with cardiovascular (CV) damage, infection, slowed linear growth, and social and intellectual development, as well as significant excess challenges in children, while kidney transplantation provides a clear survival advantage $[1 \bullet]$. Transplant graft, as well as patient outcomes, continues to improve with fine-tuned immunosuppression, better infection control, and superior CV care, with large registry studies recently reporting pediatric graft half-lives approaching 15 years [2].

The prevalence of many transplant-related comorbidities continues to decline [3]. Post-operative hospitalization stays have shortened over time, with those of the most recent transplant era (2005-2013) proving to be on average 7 days shorter than the earliest captured era (1987-1995) [3]. Acute rejection rates have fallen to an all-time low, from over $50 \%$ (1987-1991) to close to $10 \%(2007-2013)$ at 1 year after kidney transplantation [3]. Introduction of human recombinant growth hormone together with reduction of corticosteroid use in 
immunosuppression protocols has proven to complement the remarkable catch-up growth observed in children (especially those under 6 years of age) after trend toward normalization of filtering function with kidney transplantation [4-6]. Malignancy in children after kidney transplantation, previously on the rise through the early 2000s, has shown a more encouraging shift in the recent decade with a healthy reversal of the historical upward trend [3].

Surgical and medical limitations for pediatric recipients and potential donors are better recognized; very small recipients are no longer matched with donor organs from young children as this combination was previously proven to be associated with high rates of graft loss from thrombotic events [7]. The importance of vigorous immunization efforts in preparation for transplantation (particularly administration of live vaccines, i.e., against measles, mumps, rubella, and varicella), minimal size limitations (upwards of $7 \mathrm{~kg}$ ), and treatment of original renal diseases prior to and after kidney transplantation (including urologic abnormalities, the most common cause of progression to ESRD among children) is better understood and managed [8-10].

\section{Persistent challenges}

Nonetheless, providers and families caring for children with renal transplants continue to be challenged by numerous "old," longstanding, as well as "new," emerging threats to successful longevity of transplants and their pediatric recipients. Although CV morbidity and mortality are markedly reduced from pre- to post- transplant status, pediatric kidney transplant recipients remain at significant elevated risk of suffering adverse $\mathrm{CV}$ outcomes as compared to their age-matched peers, facing shortened overall life expectancy [11 $\bullet$. Recent trends indicate that $\mathrm{CV}$ disease is catching up to, and may likely replace infection as the leading cause of postkidney transplant mortality in children [3, 12]. Minimizing traditional as well as non-traditional risk factors for $\mathrm{CV}$ disease is an integral component of comprehensive pediatric renal transplant care.

Non-adherence, especially during adolescence and times of change, is a frequent and vexing cause of rejection and graft loss that has persisted through early and modern transplant eras, despite great efforts in preparation for and streamlining of transitions [13]. Such non-adherence is not necessarily limited to just unreliably taking the antirejection drugs that are needed for the lifetime of the graft (typically a calcineurin inhibitor such as tacrolimus and an antiproliferative agent such as mycophenolic acid, plus possibly corticosteroids); its implications can be particularly disastrous in this context, especially because non-adherence has now also been linked to the generation of donorspecific antibodies (DSAs, see below).

Lastly, children with glomerulopathies and glomerulonephritides as causes of ESRD, as compared to children with urologic, ischemic, or tubular diseases, continue to have worse (although overall improved) long-term graft survival, often due to recurrence of the original disease in the transplanted kidney [3].

\section{Updates}

Three novel challenges to clinical management after kidney transplantation have emerged in the last decade: (1) opportunistic viral infections (specifically BK virus, BKV), (2) acute and chronic AMR and associated circulating DSA, and (3) a dramatic increase in overweight and obesity in children after kidney transplantation as discussed in detail below. Regarding the former two (BK viral infection and AMR), much progress has been made in the development and application of diagnostic tools and therapeutic interventions, allowing for the much improved distinction of either BKV-related complications or AMR from acute cellular rejection (ACR), the "classic" immune-mediated cause of acute graft dysfunction. 


\section{BK virus}

BK virus (named after the initials of the first patient in whom it was identified) is a rather ubiquitous resident of the human urothelium after adolescence [14] and typically contained by the host's intact immune system. In immunosuppressed individuals, BKV can escape this containment and cause lower as well as upper urinary tract disease, most notably hemorrhagic cystitis in recipients of hematopoietic stem cell transplants and BKV nephritis and nephropathy in patients with kidney and other solid organ transplants [15, 16]. BKV-mediated renal damage is now clearly recognized as a distinct cause of decline in native (in non-kidney solid organ transplant recipients) and graft (in kidney recipients) function. Ideally, it is preventable and at minimum, should be recognized and contained early in its course. In kidney transplant recipients, regular plasma or serum monitoring for BKV by PCR is recommended; repeat significant elevations of BKV DNA copy numbers in the circulation are associated with a

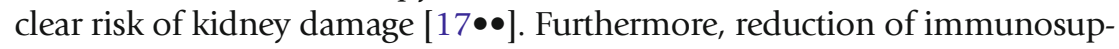
pression is recommended in patients with significant $\mathrm{BK}$ viremia and stable kidney function who are not at high immunological risk for rejection $[17 \bullet \bullet, 18]$ while the addition of antiviral therapy should be considered for those patients at increased immunological risk, i.e., where options for reduction in immunosuppression are limited. Patients with BK viremia and worsening kidney function require a kidney biopsy to guide management: if BKV nephritis is confirmed and other causes of graft dysfunction are excluded histologically, the management approach is similar to the cohort of individuals with stable kidney function. The established mainstay of therapy in the setting of unstable kidney function and absent rejection on biopsy is reduction of immunosuppression, potentially augmented by antiviral (leflunomide, cidofovir, $[17 \bullet \bullet, 18]$ ) and other (IVIG, [19]) therapies depending on the severity of BKV nephritis and kidney dysfunction [20, 21]. In fact, BK viremia and nephritis can be considered a "biomarker" of over-immunosuppression [22].

\section{BKV nephritis (too much immunosuppression) can look like cellular rejection (not enough}

Because reduction in immunosuppression is such an important intervention for the management of BKV-related disease and because rejection typically requires "opposite" therapeutic measures (i.e., increased immunosuppression), the distinction of BKV nephritis from rejection as causes of kidney transplant dysfunction is critical. Until recently, this turned out to be quite challenging at times because BKV nephritis and ACR can look extremely similar on routine histology, with both featuring tubulointerstitial inflammation and especially tubulitis as their morphological hallmark ([23], Fig. 1). If present, viral cytopathic changes can also aid in making an accurate diagnosis, and special stains for BKV are now routinely available to further facilitate this important diagnostic clarification with major implications for therapy ([23], Fig. 1). Trained observers can also detect decoy cells or even decoy cell casts in specially stained urine samples, with decoy cell casts being considered specific indicators of BK virus affecting the kidney(s) [23]. Another promising advancement along these lines, especially considering the substantial cost of PCRs and risks involved in kidney biopsies, may be the electronmicroscopic monitoring of urine samples 


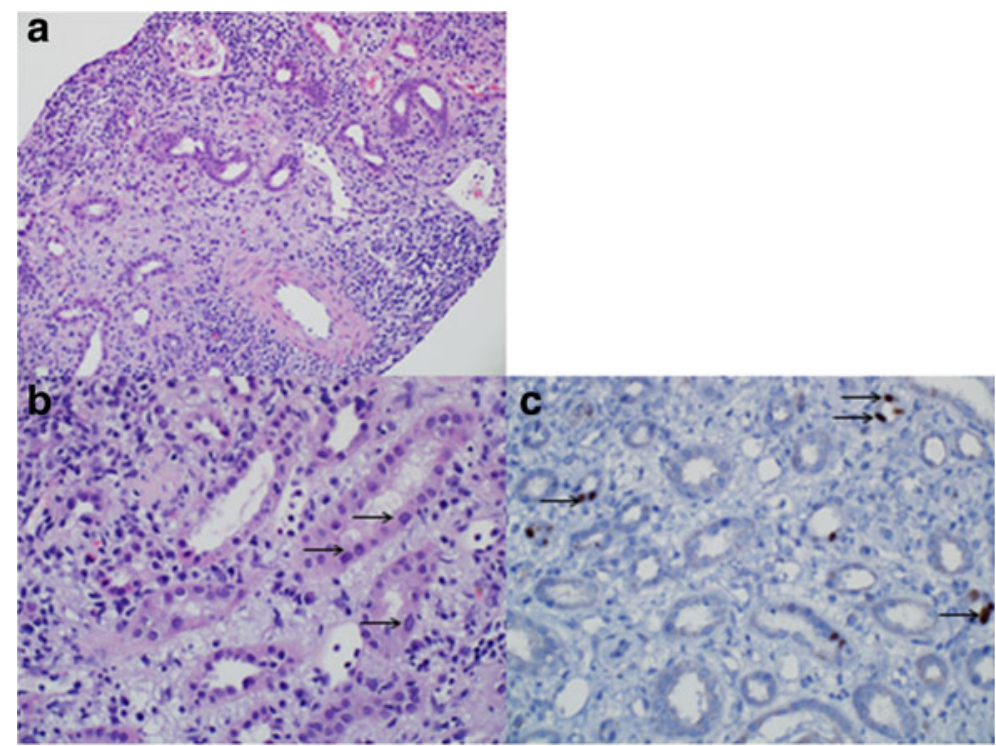

Fig. 1. Kidney transplant biopsy from a young adult recipient whose immunosuppression had been increased previously to treat an episode of acute cellular rejection (ACR). This biopsy, performed subsequent to recurrent elevations in the patient's serum creatinine as well as the de novo detection of BK viremia, again shows tubulointerstitial inflammation and tubulitis that could suggest recurrent ACR (panel $a, H \& E, 200 x$ ). However, there are now accompanying viral cytopathic changes in a number of tubular epithelial cells (arrows in panel b, H \& E, 400x), and BK immunostaining is positive (arrows in panel $\mathbf{c}$, 400x). Immunosuppression was therefore down-modulated by substituting the strong antiproliferative mycophenolic acid with leflunomide, an antiinflammatory compound that also has antiviral properties. Serum creatinine subsequently improved and BK viremia cleared, allowing eventual return to standard immunosuppression. Throughout the patient's course, there was never any evidence of antibody-mediated rejection such as donor-specific antibodies in the blood or peritubular C4d staining on biopsies (not shown). Pathological slides courtesy of Dr. Kelley Capocelli, Medical Director, Anatomic Pathology and Transfusion Medicine, Children's Hospital Colorado.

for BKV "Haufen" ("piles," [24]), although this approach still requires validation before its routine clinical applicability.

\section{Rejection can be antibody-mediated and look very different from the tubulitis seen in cellular rejection or BKV nephritis}

Regarding rejection, our historic notion was that it is a largely $\mathrm{T}$ celldriven and -executed process resulting in the infiltration and destruction of kidney tissue, predominantly tubules because of their prominent expression of donor HLA molecules serving as immunological "targets," hence, the term tubulitis as the typical morphological feature of ACR (see above). It is abundantly clear now that cellular rejection is only one of several pathways of immunological graft injury and that humoral pathways of various acuities and with variable degrees of complement involvement also exist, either separately from or jointly with cellular rejection [25•]. Humoral rejection is typically mediated by antibodies against donor tissue, explaining the term AMR. While some of these antibodies can target other molecules [25•], they are usually directed against donor HLA and thus called DSAs. Plasma monitoring for DSAs is now not only possible but recommended as routine follow-up in kidney transplant recipients [26], as their appearance, in stark contrast to 
the detection of BKV (see above), is considered a "biomarker" of underimmunosuppression, either iatrogenic or possibly related to nonadherence [26]. In the context of a new DSA, a transplant biopsy should be considered to guide further, acute management [26]. Persistent, "old" DSAs, on the other hand, may be much more ominous predictors of poor outcomes, especially if they are accompanied by chronic changes on biopsy, with transplant glomerulopathy being one of the worst manifestations of long-term damage $[25 \bullet, 27]$. The diagnosis and classification of humoral rejection has been substantially refined over the last decade or two, utilizing histological criteria as well as the presence or absence of DSAs in the circulation as important criteria. Histologically, peritubular deposition of $\mathrm{C} 4 \mathrm{~d}$, an end-product of complementmediated tissue injury, has been a hallmark of AMR [28]; it is now recognized that AMR can also occur with negative C4d staining, presumably mediated by antibodies that damage the graft tissue directly and without much complement involvement [25•]. Further characterization of circulating DSAs regarding IgG subclass identities and their relative ability to activate complement (as different such subclasses are known to differ in their propensity to bind complement) holds promise going forward to enhance our ability to gauge how pathogenic individual DSAs may be [29]. In the future, such an enhanced ability may translate into more sophisticated and graded approaches in response to the detection of DSAs and resultant refined management of established AMR.

Late, and especially chronic, AMR remains a therapeutic enigma with very few convincing management options that may improve or at least preserve graft function $[25 \bullet$, 27]. In contrast, the treatment of early, acute AMR has evolved quite nicely. Enhanced immunosuppression is the therapy mainstay, however, and not surprisingly, is largely provided by the addition of agents that are not commonly used for the prevention or management of cellular rejection. Instead, a threefold strategy involving (1) suppression of antibody production (with rituximab or a proteasome inhibitor such as bortezomib), (2) modulation of antibody effects with IVIG, and (3) removal of existing antibodies by plasmapheresis has shown to be effective in reversing acute AMR and decreasing circulating DSA loads [28].

\section{Cardiovascular disease in pediatric kidney transplantation: "obesity epidemic on steroids"}

While addressing two of the three recently evolved challenges in pediatric (and adult) kidney transplantation, i.e., BKV and DSAs/AMR, has benefitted greatly from scientific advances such as the development of molecular assays and stains for BKV, DSAs or C4d and from the development of novel therapeutic approaches, the third current challenge - the overweight/obesity/CV disease spectrum-presents a much more "societal" and largely non-immunological challenge: $\mathrm{CV}$ disease is the second most common cause of death in children after kidney transplantation [3]. Study of USRDS data demonstrates that cardiac deaths in children and young adults with ESRD are 1000 times more likely than in the general population; risk is reduced after 
transplantation, but remains markedly higher than in healthy peers $[11 \bullet$, 30•]. Moreover, in pediatric kidney transplant recipients, first CV events are often fatal $(30 \%)$ and rarely antedated by non-fatal warnings [31]. These trends may, in part, be mediated by the pre-transplant CKD/ESRD milieu that favors arterial medial vascular calcification which persists even after improvement of filtering function with transplantation [32]. Not surprisingly, American Heart Association guidelines for cardiovascular risk reduction stratify children with kidney transplants in the Tier 1 high risk category for the "development of pathological and/or clinical evidence for manifesting coronary disease before 30 years of age" [33•].

As in the general pediatric population, childhood overweight and obesity are significant health challenges in children after kidney transplantation. Optimization of nutritional status is often difficult in growing children awaiting transplant, likely influenced by multiple factors including reduced appetite (uremia), increased catabolic state (acidosis), loss of potential nutrients in dialysate, and chronic inflammation $[4,34]$. Weight gain after kidney transplantation, indiscriminant of recipient age, may well be an appropriate result of improved appetite and reversal of the uremic milieu. In fact, in children, catch-up growth with concomitant weight gain is anticipated, especially in the first year after transplantation [35]. However, weight gain commonly exceeds expectations of linear catch-up growth [3], and prevalence of obesity and overweight in children after kidney transplantation often exceed figures reflected in the general population [36-38]. Not only does higher BMI during childhood raise the risk for development of $\mathrm{CV}$ disease during adulthood, obesity at the time of or subsequent to kidney transplant is an established risk factor for poor graft and overall patient survival outcomes [38-40]. Moreover, pediatric kidney transplant centers are often unpredictable and unreliable in the management of the CV health of our patients, although quality improvement (QI) and related tools have been shown to be useful in improving this [41-43].

\section{Conclusion}

Kidney transplantation remains the best therapeutic option for children with ESRD. Progress has been made against many of the known challenges that are being posed by this treatment modality, i.e., chronic immunosuppression and increased CV risk. Out of these known challenges, several novel questions, insights, and interventions have evolved the following: (1) The recognition of BK virus-related kidney and urinary tract disease as a biomarker of over-immunosuppression; (2) The emergence of tools to better recognize, characterize, and manage AMR as a biomarker of under-immunosuppression and possibly of non-adherence; and (3) The dramatic degree of CV disease risk in kidney transplant recipients in the contexts of the obesity epidemic affecting our whole society and of the dismal lack of effectiveness of our health care system in managing this risk despite the ample availability of diagnostic and therapeutic interventions. Accordingly, innovative approaches to this 
problem are sorely needed, such as QI-driven strategies and learning and care networks.

\section{Compliance with Ethical Standards}

Conflict of Interest

Margret Bock and Jens Goebel declare that they have no conflict of interest.

Human and Animal Rights and Informed Consent

This article does not contain any studies with human or animal subjects performed by any of the authors.

\section{References and Recommended Reading}

Papers of particular interest, published recently, have been

highlighted as:

- Of importance

$\bullet \quad$ Of major importance

1.• Wolfe RA, Ashby VB, Milford EL, et al. Comparison of mortality in all patients on dialysis, patients on dialysis awaiting transplantation, and recipients of a first cadaveric transplant. N Engl J Med. 1999;341:1725-30.

Seminal analysis first demonstrating the survival benefit of kidney transplantation over all forms of dialysis.

2. Organ Procurement and Transplantation Network (OPTN) and Scientific Registry of Transplant Recipients (SRTR). OPTN/SRTR 2012 annual data report. Rockville, MD: Department of Health and Human Services, Heath Resources and Services Administration; 2014.

3. North American Pediatric Renal Transplant Cooperative Study (NAPRTCS). Annual transplant report. MD: Rockville; 2014. Available from http://naprtcs.org.

4. Laster ML, Fine RN. Growth following solid organ transplantation in childhood. Pediatr Transplant. 2014;19:134-41.

5. Webb NJ, Douglas SE, Rjai A, et al. Corticosteroid-free kidney transplantation improves growth. Transplantation. 2015;99:1178-85.

6. Sarwal MM, Ettenger RB, Dharnidharka V, et al. Complete steroid avoidance is effective and safe in children with renal transplants: a multi-center trial with threeyear follow-up. Am J Transplant. 2012;12:2719-29.

7. Harmon WE, Alexander SR, Tejani A, et al. The effect of donor age on graft survival in pediatric cadaver renal transplant recipients-a report of the North American Pediatric Renal Transplant Cooperative Study. Transplantation. 1992;54:232-7.

8. Neu AM, Fivush BA. Recommended immunization practices for pediatric renal transplant recipients. Pediatr Transplant. 1998;2:263-9.
9. Dharnidharka VR, Agodoa LY, Abbott KC. Effects of urinary tract infection on outcomes after renal transplantation in children. Clin J Am Soc Nephrol. 2007;2:100-6.

10. Sarwal MM, Cecka JM, Millan MT, et al. Adult-size kidneys without acute tubular necrosis provide exceedingly superior long-term graft outcomes for infants and small children: a single center and UNOS analysis. Transplantation. 2000;70:1728-36.

11. Parekh RS, Carroll CE, Wolfe RA, et al. Cardiovascular mortality in children and young adults with end stage renal disease. J Pediatr. 2002;141:191-7.

An excellent population analysis that confirms that children with ESRD, like adults, are at dramatically increased risk for cardiac death and morbidity, as compared to healthy peers.

12. Foster BJ, Dahhou M, Zhang X, et al. Change in mortality risk of time in young kidney transplant recipients. Am J Transplant. 2011;11:2432-42.

13. Bell LE, Bartosh SM, Davis CL, et al. Adolescent transition to adult care in solid organ transplantation: a consensus conference report. Am J Transplant. 2008;8:2230-42.

14. Dalianis T, Hirsch HH. Human poliomaviruses in disease and cancer. Virology. 2013;437:63-72.

15. Haines HL, Laskin BL, Goebel J, et al. Blood and not urine BK viral load predicts renal outcome in children with hemorrhagic cystitis following hematopoietic stem cell transplantation. Biol Blood Marrow Transplant. 2011;17:1512-9.

16. Hirsch HH, Randhawa P, the AST Infectious Disease Community of Practice. BK virus in solid organ transplant recipients. Am J Transplant. 2009;9 Suppl 4:S136-46. 
17.• Kidney Disease: Improving Global Outcomes (KDIGO) Transplant Work Group. KDIGO clinical practice guidelines for the care of kidney transplant recipients. Am J Transplant. 2009;9 Suppl 3:S1-157.

The "go-to" resource for post-kidney transplant care-even with some specific information for pediatric recipients.

18. Johnston O, Jaswal J, Gill JS, et al. Treatment of polyomavirus infection in kidney transplant recipients: a systematic review. Transplantation. 2010;89(9):105770.

19. Randhawa P, Pastrana DV, Zeng G, et al. Commercially available immunoglobulins contain virus neutralizing antibodies against all major genotypes of polyomavirus BK. Am J Transplant. 2015;15(4):1014-20.

20. Araya CE, Garin EH, Neiberger RE, et al. Leflunomide therapy for BK virus allograft nephropathy in pediatric and young adult kidney transplant recipients. Pediatr Transplant. 2010;14:145-50.

21. Araya CE, Lew JF, Fennell RS, et al. Intermediate dose cidofovir does not cause additive nephrotoxicity in BK virus allograft nephropathy. Pediatr Transplant. 2008;12:790-5.

22. Borni-Duval C, Caillard S, Olagne J, et al. Risk factors for BK virus infection in the era of therapeutic drug monitoring. Transplantation. 2013;95:1498-505.

23. Ramos E, Drachenberg CB, Wali R, Hirsch HH. The decade of polyomavirus BK-associated nephropathy: state of affairs. Transplantation. 2009;87:621-30.

24. Laskin BL, Singh HK, Beier UH et al. The urinary polyomavirus Haufen test non-invasively detects kidney injury in children and young adults after allogeneic hematopoietic cell transplantation. Transplantation (in press).

25. Loupy A, Hill GS, Jordan SC. The impact of donorspecific anti-HLA antibodies on late kidney allograft failure. Nat Rev Nephrol. 2012;8:348-57.

An excellent and detailed review of what we currently know about DSAs in kidney transplantation.

26. Tait BD, Suesal C, Gebel HM, et al. Consensus guidelines on the testing and clinical management issues associated with HLA and non-HLA antibodies in transplantation. Transplantation. 2013;95:19-47.

27. David-Neto E, Prado E, Beutel A, et al. C4d-positive chronic rejection: a frequent entity with a poor outcome. Transplantation. 2007;84:1391-8.

28. Claes DJ, Yin H, Goebel J. Protective immunity and use of bortezomib for antibody-mediated rejection in a pediatric kidney transplant recipient. Pediatr Transplant. 2014;18:E100-5.

29. Lefaucheur C, Viglietti D, Bentlejewski C, et al. IgG donor-specific anti-human HLA antibody subclasses and kidney allograft antibody-mediated injury. J Am Soc Nephrol. 2016;27:293-304.

30. Schmidt B, Thurn-Valsassina D, Dizowa A, et al. Longitudinal evaluation of cardiovascular risk after pediatric kidney transplantation. J Hypertens. 2015;33:e16.

Further resounding evidence that kidney transplantation lowers long-term cardiovascular risk in children and young adults.
31. Khoshy SM, Guttman A, Herebt D, et al. Incidence and risk factor for cardiovascular events and death in pediatric renal transplant patients: a single center long-term outcome study. Pediatr Transplant. 2009;13:1027-33.

32. Schroff R, Quinlan C, Mitsnefes M. Uraemic vasculopathy in children with chronic kidney disease: prevention or damage limitation? Pediatr Transplant. 2011;26:853-65.

33. Kavey RE, Allada V, Daniels SR, et al. Cardiovascular risk reduction in high-risk pediatric patients: a scientific statement from the American Heart Association Expert Panel ON Population and Prevention Science; The Councils on Cardiovascular Disease in the Young, Epidemiology and Prevention, Nutrition, Physical Activity and Metabolism, High Blood Pressure Research, Cardiovascular Nursing, and the Kidney in Heart Disease; and The Interdisciplinary Working Group on Quality of Care and Outcomes Research: endorsed by The American Academy of Pediatrics. Circulation. 2006; 114:2710-38.

A mainstay resource by the American Heart Association on cardiovascular risk in high-risk pediatric patients.

34. Fine RN. Etiology and treatment of growth retardation in children with chronic kidney disease and end-stage renal disease: a historical perspective. Pediatr Nephrol. 2010;25:725-32.

35. Fine RN. Management of growth retardation in pediatric recipients of renal allografts. Nat Clin Pract Nephrol. 2007;3:318-24.

36. Plumb LA, Pitcher D, Tse Y, et al. Longitudinal changes in body mass index following renal transplantation in UK children. Nephrol Dial Transplant. 2014;29:196-203.

37. Foster BJ, Martz K, Gowrishankar M, et al. Weight and height changes and factors associated with greater weight and height gains after pediatric renal transplantation: a NAPRTCS study. Transplantation. 2010;89:1103-12.

38. Mitsnefes M, Khoury P, McEnery PT. Body mass index and allograft function in pediatric renal transplantation. Pediatr Nephrol. 2002;17:535-9.

39. Baker JL, Olsen LW, Sorensen TI. Childhood body mass index and the risk of coronary heart disease in adulthood. N Engl J Med. 2007;357:2329-37.

40. Meier-Kriesche HU, Arndorfer JA, Kaplan B. The impact of body mass index on renal transplant outcomes: a significant independent risk factor for graft failure and patient death. Transplantation. 2002;72:70-4.

41. Wilson AC, Greenbaum LA, Barletta GM, et al. High prevalence of the metabolic syndrome and associated left ventricular hypertrophy in pediatric renal transplant recipients. Pediatr Transplant. 2010;14:52-60.

42. Hooper DK, Williams JC, Carle AC, et al. The quality of cardiovascular disease care for adolescents with kidney disease: a Midwest Pediatric Nephrology Consortium Study. Pediatr Nephrol. 2013;28:939-49.

43. Hooper DK, Kirby C, Margolis P, Goebel J. Reliable individualized monitoring improves cholesterol control in kidney transplant recipients. Pediatrics. 2013;131(4):e1271-9. 